\title{
Discovery of the two "wings" of the Kookaburra complex in VHE $\gamma$-rays with HESS
}

\author{
F. Aharonian ${ }^{1}$, A. G. Akhperjanian ${ }^{2}$, A. R. Bazer-Bachi ${ }^{3}$, M. Beilicke ${ }^{4}$, W. Benbow ${ }^{1}$, D. Berge ${ }^{1}$, K. Bernlöhr $^{1,5}$,
} C. Boisson ${ }^{6}$, O. Bolz ${ }^{1}$, V. Borrel ${ }^{3}$, I. Braun ${ }^{1}$, A. M. Brown ${ }^{7}$, R. Bühler ${ }^{1}$, I. Büsching ${ }^{8}$, S. Carrigan ${ }^{1}$, P. M. Chadwick ${ }^{7}$, L.-M. Chounet ${ }^{9}$, R. Cornils ${ }^{4}$, L. Costamante ${ }^{1,22}$, B. Degrange ${ }^{9}$, H. J. Dickinson ${ }^{7}$, A. Djannati-Atai ${ }^{10}$, L. O'C. Drury ${ }^{11}$, G. Dubus $^{9}$, K. Egberts ${ }^{1}$, D. Emmanoulopoulos ${ }^{12}$, P. Espigat ${ }^{10}$, F. Feinstein ${ }^{13}$, E. Ferrero ${ }^{12}$, A. Fiasson ${ }^{13}$, G. Fontaine ${ }^{9}$, S. Funk ${ }^{5}$, S. Funk ${ }^{1}$, M. Füßling ${ }^{5}$, Y. A. Gallant ${ }^{13}$, B. Giebels ${ }^{9}$, J. F. Glicenstein ${ }^{14}$, P. Goret ${ }^{14}$, C. Hadjichristidis ${ }^{7}$, D. Hauser ${ }^{1}$, M. Hauser ${ }^{12}$, G. Heinzelmann ${ }^{4}$, G. Henri ${ }^{15}$, G. Hermann ${ }^{1}$, J. A. Hinton ${ }^{1,12}$, A. Hoffmann ${ }^{16}$, W. Hofmann ${ }^{1}$, M. Holleran ${ }^{8}$, D. Horns ${ }^{16}$, A. Jacholkowska ${ }^{13}$, O. C. de Jager ${ }^{8}$, E. Kendziorra ${ }^{16}$, B. Khélifi ${ }^{9,1}$, Nu. Komin ${ }^{13}$, A. Konopelko ${ }^{5}$, K. Kosack ${ }^{1}$, I. J. Latham ${ }^{7}$, R. Le Gallou ${ }^{7}$, A. Lemière ${ }^{10}$, M. Lemoine-Goumard ${ }^{9}$, T. Lohse ${ }^{5}$, J. M. Martin 6 , O. Martineau-Huynh ${ }^{17}$, A. Marcowith ${ }^{3}$, C. Masterson ${ }^{1,22}$, G. Maurin ${ }^{10}$, T. J. L. McComb ${ }^{7}$, M. de Naurois ${ }^{17}$, D. Nedbal ${ }^{18}$, S. J. Nolan ${ }^{7}$, A. Noutsos ${ }^{7}$, K. J. Orford ${ }^{7}$, J. L. Osborne ${ }^{7}$, M. Ouchrif ${ }^{17,22}$, M. Panter ${ }^{1}$, G. Pelletier ${ }^{15}$, S. Pita ${ }^{10}$, G. Pühlhofer ${ }^{12}$, M. Punch ${ }^{10}$, B. C. Raubenheimer ${ }^{8}$, M. Raue ${ }^{4}$, S. M. Rayner ${ }^{7}$, A. Reimer ${ }^{19}$, O. Reimer ${ }^{19}$, J. Ripken ${ }^{4}$, L. Rob ${ }^{18}$, L. Rolland ${ }^{14}$, G. Rowell ${ }^{1}$, V. Sahakian ${ }^{2}$, A. Santangelo ${ }^{16}$, L. Saugé ${ }^{15}$, S. Schlenker ${ }^{5}$, R. Schlickeiser ${ }^{19}$, R. Schröder ${ }^{19}$, U. Schwanke ${ }^{5}$, S. Schwarzburg ${ }^{16}$, A. Shalchi ${ }^{19}$, H. Sol ${ }^{6}$, D. Spangler ${ }^{7}$, F. Spanier ${ }^{19}$, R. Steenkamp ${ }^{20}$, C. Stegmann ${ }^{21}$, G. Superina ${ }^{9}$, J.-P. Tavernet ${ }^{17}$, R. Terrier $^{10}$, C. G. Théoret ${ }^{10}$, M. Tluczykont ${ }^{9,22}$, C. van Eldik ${ }^{1}$, G. Vasileiadis ${ }^{13}$, C. Venter ${ }^{8}$, P. Vincent ${ }^{17}$, H. J. Völk ${ }^{1}$, S. J. Wagner ${ }^{12}$, and M. Ward ${ }^{7}$

(Affiliations can be found after the references)

Received 26 April 2006 / Accepted 12 June 2006

\section{ABSTRACT}

\begin{abstract}
Aims. Search for Very High Energy $\gamma$-ray emission in the Kookaburra complex through observations with the HESS array. Methods. Stereoscopic imaging of Cherenkov light emission of the $\gamma$-ray showers in the atmosphere is used for the reconstruction and selection of the events to search for $\gamma$-ray signals. Their spectrum is derived by a forward-folding maximum likelihood fit.

Results. Two extended $\gamma$-ray sources with an angular (68\%) radius of 3.3-3.4' are discovered at high $(>13 \sigma)$ statistical significance: HESS J1420-607 and HESS J1418-609. They exhibit a flux above $1 \mathrm{TeV}$ of $\left(2.97 \pm 0.18_{\text {stat }} \pm 0.60_{\text {sys }}\right) \times 10^{-12}$ and $\left(2.17 \pm 0.17_{\text {stat }} \pm 0.43_{\text {sys }}\right) \times$ $10^{-12} \mathrm{~cm}^{-2} \mathrm{~s}^{-1}$, respectively, and similar hard photon indices $\sim 2.2$. Multi-wavelength comparisons show spatial coincidence with the wings of the Kookaburra. Two pulsar wind nebulæ candidates, K3/PSR J1420-6048 and the Rabbit, lie on the edge of the HESS sources.

Conclusions. The two new sources confirm the non-thermal nature of at least parts of the two radio wings which overlap with the $\gamma$-ray emission and establish their connection with the two X-ray pulsar wind nebulæ candidates. Given the large point spread function of EGRET, the unidentified source(s) 3EG J1420-6038/GeV J1417-6100 could possibly be related to either or both HESS sources. The most likely explanation for the Very High Energy $\gamma$-rays discovered by HESS is inverse Compton emission of accelerated electrons on the Cosmic Microwave Background near the two candidate pulsar wind nebulæ, K3/PSR J1420-6048 and the Rabbit. Two scenarios which could lead to the observed large ( 10 pc) offset-nebula type morphologies are briefly discussed.
\end{abstract}

Key words. ISM: general - gamma rays: observations - radiation mechanisms: non-thermal

\section{Introduction}

The complex of compact and extended radio/X-ray sources called Kookaburra, after the name of the Australian bird (Roberts et al. 1999), spans over about one square degree along the Galactic plane around $l=313.4^{\circ}$. It has been extensively studied in the search for counterparts to the unidentified EGRET source (or sources, see Sect. 3.3) 3EG J1420-6038/GeV J1417-6100 (Hartman et al. 1999; Lamb \& Macomb 1997).

Radio images of this region have revealed a large circular thermal shell with a broad wing to the North-East and a narrower wing to the South-West. Diffuse X-ray emission in the wings and point sources have been discovered through ASCA observations (Roberts et al. 1999, 2001b), followed by higher resolution imaging with XMM-Newton and Chandra (Ng et al. 2005). A $3^{\prime} \mathrm{X}$-ray/radio nebula in the North-Eastern wing contains a young $\left(\tau_{\mathrm{c}}=1.3 \times 10^{4} \mathrm{yr}\right)$ and very energetic radio pulsar $\left(\dot{E}=1.0 \times 10^{37} \mathrm{erg} / \mathrm{s}\right.$ ), PSR J1420-6048 (D'Amico et al. 2001), and has been proposed as a candidate pulsar wind nebula (PWN) counterpart to the EGRET source(s). A brighter nebula, G313.1+0.1, called the "Rabbit" (Roberts et al. 1999), lying in the South-Western wing and exhibiting extended hard X-ray emission has been proposed as a plausible PWN contributing also to the $\gamma$-ray emission detected by EGRET.

In this paper, observations of the Kookaburra region with the HESS (High Energy Stereoscopic System) telescopes and the discovery of two Very High Energy (VHE) $\gamma$-ray sources coinciding with its wings are reported. HESS is an array of four imaging atmospheric Cherenkov telescopes located in the 
Khomas Highland of Namibia (Hinton 2004). Each HESS telescope has a mirror area of $107 \mathrm{~m}^{2}$ (Bernlöhr et al. 2003) and a total field of view of $5^{\circ}$ (Vincent et al. 2003). The system is run in a coincidence mode (Funk et al. 2004) requiring at least two of the four telescopes to have triggered in each event. The HESS instrument has an angular resolution of $\sim 5^{\prime}$ per event and a point-source sensitivity of $<2.0 \times 10^{-13} \mathrm{~cm}^{-2} \mathrm{~s}^{-1}$ (1\% of the flux from the Crab Nebula above $1 \mathrm{TeV}$ ) for a $5 \sigma$ detection in a 25 h observation.

Section 2 describes the HESS observations, data reduction and results. In Sect. 3 multi-wavelength comparisons are made and in the following section the interpretation of data together with possible associations for the new HESS sources are discussed.

\section{HESS observations}

The first observations of the Kookaburra region took place in a survey of the Galactic plane in the range of Galactic longitudes $300^{\circ}<l<330^{\circ}$ and Galactic latitudes $-3^{\circ}<b<3^{\circ}$. This survey, which was taken between April and July 2005, represents the extension of the HESS 2004 survey of the inner Galaxy (Aharonian et al. 2005a, 2006a) toward lower Galactic longitudes. The detection of a $\gamma$-ray signal from the Kookaburra region triggered re-observations between May and August 2005 for $11.1 \mathrm{~h}$ in pointed observations alternating at an offset of $0.7^{\circ}$ in declination around a central position in the Kookaburra $\left(14^{\mathrm{h}} 20^{\mathrm{m}} 0^{\mathrm{s}},-60^{\mathrm{d}} 45^{\prime}\right)$. The average zenith angle of observations was $35.3^{\circ}$. The dead-time corrected data set amounts to $18.1 \mathrm{~h}$ within $2^{\circ}$ of the central position in the Kookaburra.

After calibration, the standard HESS event reconstruction scheme was applied to the data (see Aharonian et al. 2005b, for details). Cuts on the scaled width and length of images (optimised on $\gamma$-ray simulations and off-source data) are used to suppress the hadronic background. As previously described (Aharonian et al. 2006a), two different sets of image size cuts are applied: to study the morphology of a source and achieve a maximum signal-to-noise ratio for a weak source with a hard spectrum, a rather tight cut on the number of photoelectrons (p.e.) in the image of 200 p.e. is applied. For the spectral analysis this image size cut is loosened to 80 p.e. to achieve a maximum coverage in energy, resulting in a spectral analysis threshold of $300 \mathrm{GeV}$ for the dataset described here. Different methods for deriving a background estimate as described in Hinton et al. (2005) are applied. For the spectral analysis, the background is usually taken from positions in the field of view with the same offset from the pointing direction as the source region to obliviate the need for corrections concerning the radial dependence of the background acceptance. The background estimate for each position in the two-dimensional sky map is taken from a ring of mean radius $0.7^{\circ}$ and an area seven times that of the on-source region. In all background estimation methods, known $\gamma$-ray emitting regions are excluded from the background regions to avoid $\gamma$-ray contamination of the background estimate (after iterations for newly discovered sources). It should be noted that consistent results are achieved with different background estimation techniques. Figure 1 shows a smoothed excess counts map of the Kookaburra region along with contours that correspond to $5 \sigma, 7.5 \sigma$, and $10 \sigma$ significance levels.

Two sources of very high energy $\gamma$-rays are apparent in this map at high statistical significance. The stronger of the two sources, HESS J1420-607, extends to the North of the energetic pulsar PSR J1420-6048. Slices in different directions through

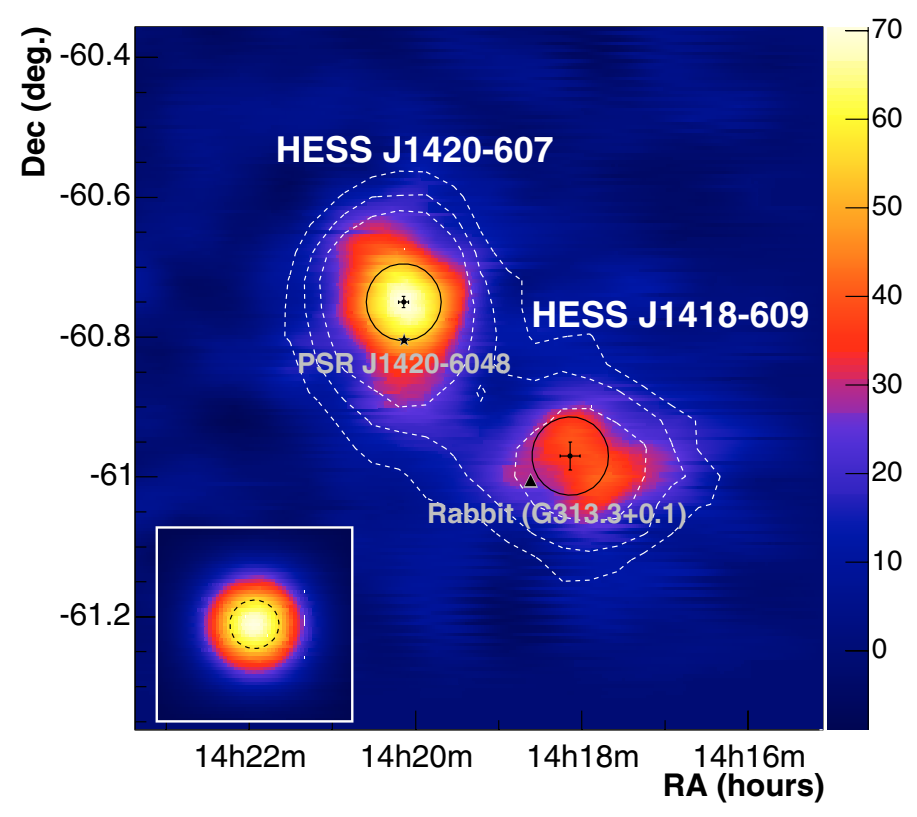

Fig. 1. Smoothed excess map of the $1^{\circ} \times 1^{\circ}$ field of view in the Kookaburra region. The unbinned excess map has been smoothed with a Gaussian of width $2^{\prime}$. In the bottom left corner the point-spread function of this dataset smoothed in the same way is shown (along with the smoothing radius as a black dashed line). The white contours denote the $5 \sigma, 7.5 \sigma$ and $10 \sigma$ significance levels (with the outermost being the $5 \sigma$ contour), derived with a point source integration radius of $0.1^{\circ}$. The position of the pulsar PSR J1420-6048 is marked with a star, the position of the rabbit $(\mathrm{G} 313.3+0.1)$ is marked with a black and white triangle. The best fit positions of the two sources are marked with error crosses, the best-fit extensions are given as black circles. There is no significant evidence for a connecting bridge beyond what is expected from the Gaussian source shape convoluted with the point spread function of the instrument.

the source show a symmetric profile with consistent extensions. Therefore, the assumption of a radially symmetric Gaussian emission region $\left(\rho \propto \exp \left(-\theta^{2} / 2 \sigma^{2}\right)\right.$ with $\sigma^{2}=\sigma_{\mathrm{PSF}}^{2}+\sigma_{\text {source }}^{2}$, $\sigma_{\mathrm{PSF}}$ characterizing the point spread function) seems well justified. With this assumption an intrinsic extension of $\sigma_{\text {source }}=$ $3.3^{\prime} \pm 0.5^{\prime}$ is derived. The best-fit position for the centre of the excess lies at $14^{\mathrm{h}} 20^{\mathrm{m}} 9^{\mathrm{s}} \pm 4^{\mathrm{s}},-60^{\mathrm{d}} 45^{\prime} 36^{\prime \prime} \pm 32^{\prime \prime}$. The slightly less bright second source, HESS J1418-609, at a distance of $\sim 33^{\prime}$ to the South-West from HESS J1420-607 extends to the West of the Rabbit, at $14^{\mathrm{h}} 18^{\mathrm{m}} 4^{\mathrm{s}} \pm 7^{\mathrm{s}},-60^{\mathrm{d}} 58^{\prime} 31^{\prime \prime} \pm 35^{\prime \prime}$ at a distance of $8.2^{\prime}$ to the position of the Rabbit. The best fit extension of HESS J1418-609 is $\sigma_{\text {source }}=3.4^{\prime} \pm 0.6^{\prime}$. Fitting an elongated Gaussian shape to this source yields a semi-major axis of $4.9^{\prime} \pm 1.5^{\prime}$ and a semi-minor axis of $2.7^{\prime} \pm 0.7^{\prime}$ at a position angle of $46.2^{\circ} \pm 20.4^{\circ}$ (major axis, North to East). Using the positions derived above and applying a cut on the reconstructed angular distance of $\gamma$-ray candidate events from this best-fit position of $\theta<0.16^{\circ}$, yields a statistical (pre-trial) significance of $15.2 \sigma$ at an excess of $692 \pm 26$ events for HESS J1420-607 and a statistical (pre-trial) significance of $13.2 \sigma$ and an excess of $576 \pm 24$ events for HESS J1418-609. The post-trial significances differ by less than $0.5 \sigma$. There is no significant evidence for a connecting bridge beyond what is expected from the Gaussian source shape convoluted with the point spread function of the instrument. Using the fit function, the contamination of HESS J1420-607 in the integration circle of HESS J1418-609 was estimated to be $3 \%$. 


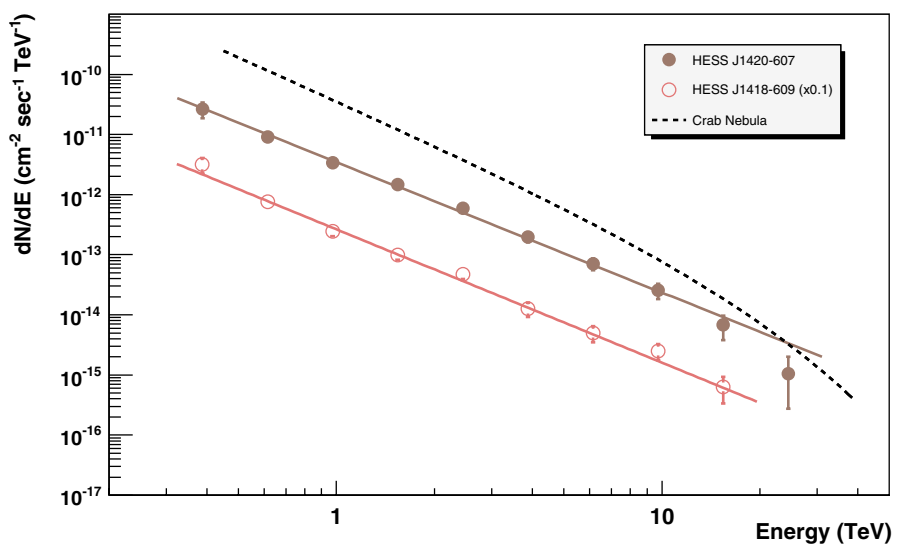

Fig. 2. Reconstructed differential VHE $\gamma$-ray spectrum of HESS J1420-607 (full circles) and HESS J1418-609 (open circles) along with power law fits to the two spectra. The flux points for HESS J1418-609 have been scaled by a factor of 0.1 for ease of viewing. The fit to the HESS J1420-607 data results in a photon index of $2.17 \pm 0.06_{\text {stat }} \pm 0.1_{\text {sys }}$ with a flux normalisation at $1 \mathrm{TeV}$ of $\left(3.48 \pm 0.20_{\text {stat }} \pm 0.70_{\text {sys }}\right) \times 10^{-12} \mathrm{~cm}^{-2} \mathrm{~s}^{-1} \mathrm{TeV}^{-1}$. The power-law fit for HESS J1418-609 yields a photon index of $2.22 \pm 0.08_{\text {stat }} \pm 0.1_{\text {sys }}$ and a flux normalisation of $\left(2.64 \pm 0.20_{\text {stat }} \pm 0.53_{\text {sys }}\right) \times 10^{-12} \mathrm{~cm}^{-2} \mathrm{~s}^{-1} \mathrm{TeV}^{-1}$. The dashed line shows the Crab nebula spectrum as measured by HESS (Aharonian et al. 2006c).

The flux of HESS J1420-607 and of HESS J1418-609 have been determined both within a radius of $0.16^{\circ}$, to avoid any overlap in the integration regions for the two sources. The effective areas used in the determination of the energy spectra assume full containment of the source in the integration region. The background has been extracted from regions distributed on a ring with the same radius and the same offset from the pointing direction as the integration region. The energy estimation algorithm takes into account the optical efficiency change with time (characteristic timescale of years as monitored by muon images) as described in Aharonian et al. (2006c). The energy spectra of the two sources have been derived using a forward-folding maximum likelihood fit (Piron et al. 2002) and are very similar, as seen in Fig. 2. The energy spectrum of HESS J1420-607 can be fitted with a power-law with a photon index of $2.17 \pm 0.06_{\text {stat }} \pm 0.1_{\text {sys }}$ and a flux above $1 \mathrm{TeV}$ of $\left(2.97 \pm 0.18_{\text {stat }} \pm 0.60_{\text {sys }}\right) \times 10^{-12} \mathrm{~cm}^{-2} \mathrm{~s}^{-1}$ (corresponding to $13.0 \%$ of the flux from the Crab nebula above that energy). The photon index for HESS J1418-609 has a similar value of $2.22 \pm 0.08_{\text {stat }} \pm 0.1_{\text {sys }}$, the flux of this source above $1 \mathrm{TeV}$ has a value of $\left(2.17 \pm 0.17_{\text {stat }} \pm 0.43_{\text {sys }}\right) \times 10^{-12} \mathrm{~cm}^{-2} \mathrm{~s}^{-1}$ (corresponding to $9.6 \%$ of the Crab flux above that energy).

\section{Multi-wavelength search for counterparts}

To search for counterparts, published multi-wavelength data have been selected and are overlaid on HESS excess maps in the two panels of Fig. 3. The left panel shows radio contours from Australia Telescope Compact Array (ATCA, $20 \mathrm{~cm}$ high resolution image) and labels of relevant radio sources (Roberts et al. 1999). Black circles show the approximate extension of the two candidate PWNe in Kookaburra, K3/PSR J1420-6048 (3') and the Rabbit (5') in X-rays ( $\mathrm{Ng}$ et al. 2005). In the right panel, both 3EG J1420-6038 and GeV J1417-6100, are shown (confidence contours and error box, respectively) although they are not independent sources (see Sect. 3.3). X-ray data contours are taken from ASCA/GIS (Roberts et al. 2001a).

\subsection{The NE wing and K3/PSR J1420-6048}

HESS J1420-607 is in positional coincidence with the NorthEastern radio wing of Kookaburra, G313.3+0.6, or K2, which has a rectangular $\sim 12^{\prime} \times 8^{\prime}$ shape (at a minimum emission level of $4 \mathrm{mJy} /$ beam). K2 has a total flux density at $20 \mathrm{~cm} S_{20 \mathrm{~cm}} \sim$ 1 Jy, with a slight enhancement, labeled K3, of $\sim 20 \mathrm{mJy}$ around PSR J1420-6048 (Roberts et al. 1999). This young $68.2 \mathrm{~ms}$ pulsar (D'Amico et al. 2001) which shows a high spin-down luminosity of $\dot{E}=1.0 \times 10^{37} \mathrm{erg} / \mathrm{s}$, lies to the South of HESS J1420-607 at an angular distance of $\sim 3.1^{\prime}$. Given the dispersion measure for the pulsar, the Cordes \& Lazio (2002) Galactic electron density model implies a distance $d=5.6 \pm$ $0.8 \mathrm{kpc}$, closer than its initial estimated distance, $d \approx 7.7 \pm$ $1.1 \mathrm{kpc}$ based on Taylor \& Cordes (1993). Observations with ASCA and Chandra have revealed a rather hard nebular X-ray emission around PSR J1420-6048 extending to a radius $\sim 6^{\prime}$, e.g., Roberts et al. (2001a) report a $2-10 \mathrm{keV}$ ASCA flux of $4.8 \times 10^{-12} \mathrm{erg} / \mathrm{cm}^{2} \mathrm{~s}^{-1}$ with a power-law index $\Gamma=1.4 \pm 0.4$ for a fitted column density $N_{\mathrm{H}} \sim 1.8 \times 10^{22} \mathrm{~cm}^{-2}$.

The radio spectral indices of $\mathrm{K} 3$ and $\mathrm{K} 2$ are poorly constrained ( 20 to $36 \mathrm{~cm}$ : $\alpha_{20 / 36}=-0.4 \pm 0.5$ and $\alpha_{20 / 36}=-0.2 \pm 0.2$, respectively, Roberts et al. 1999), but are rather hard and compatible with typical PWNe $\left(-0.3 \lesssim \alpha_{20 / 36} \lesssim-0.1\right)$. The nonthermal nature of at least parts of $\mathrm{K} 2$ is supported by the lack of correlation with infrared emission, as well as by the X-ray hard diffuse features and now established by the TeV emission detected by HESS Ng et al. (2005) have proposed K3 as a candidate PWN and a possible counterpart to GeV 1417-6100, although the nebula detected by Chandra in the inner part of $\mathrm{K} 3$ is unexpectedly faint.

\subsection{The SW radio wing and the Rabbit nebula}

HESS J1418-609 coincides spatially with the narrower $\sim 15^{\prime} \times$ 4 ' radio wing to the South-West, labelled K4, while the Rabbit nebula, or G313.3+0.1, lies on its Eastern edge at a distance of $8.2^{\prime}$ to the fitted $\mathrm{TeV}$ position. $\mathrm{K} 4$ and the Rabbit nebula have each a radio flux of $S_{20 \mathrm{~cm}} \sim 400 \mathrm{mJy}$, with non-thermal spectral indices $(13$ to $36 \mathrm{~cm})$ of $-1.2 \pm 0.5$ and $-0.25 \pm 0.1$, respectively (Roberts et al. 1999).

In X-rays, the Rabbit nebula is brighter than $\mathrm{K} 3$, exhibiting for example in ASCA a $2-10 \mathrm{keV}$ flux of $7.33 \times$ $10^{-12} \mathrm{erg} / \mathrm{cm}^{2} \mathrm{~s}^{-1}$ (Roberts et al. 2001a), and a power-law index in the range 1.5-1.9. Two sources, labelled R1 and R2 (spaced by $42.5^{\prime \prime}$ ), were resolved by Chandra observations within the diffuse emission which extends over an area of radius $\sim 7^{\prime}(\mathrm{Ng}$ et al. 2005). These authors report a very marginal detection (chance probability of 0.02) of X-ray pulsations from the fainter source, R2, at a period of $108 \mathrm{~ms}$ and propose it as a plausible pulsar for the candidate Rabbit PWN. Tentative estimates of the pulsar spin-down luminosity, $\log (\dot{E}) \sim 36.7$ to $37.5 \mathrm{erg} / \mathrm{s}$, age $\tau=1.6 \mathrm{kyr}$ and distance, $d \sim 5 \mathrm{kpc}$ are also proposed in $\mathrm{Ng}$ et al. (2005).

\subsection{The EGRET data}

The original $\gamma$-ray source detected by EGRET in the Kookaburra region, 2EG J1412-6211, was reported in the second catalog (Thompson et al. 1995), based on the first and second year of CGRO operation. During phase 3 a new source, 2EGS J1418-6049, was detected and published in the supplement to the second EGRET catalog (Thompson et al. 1996). With the inclusion of phase 4 data, as well as due to the 

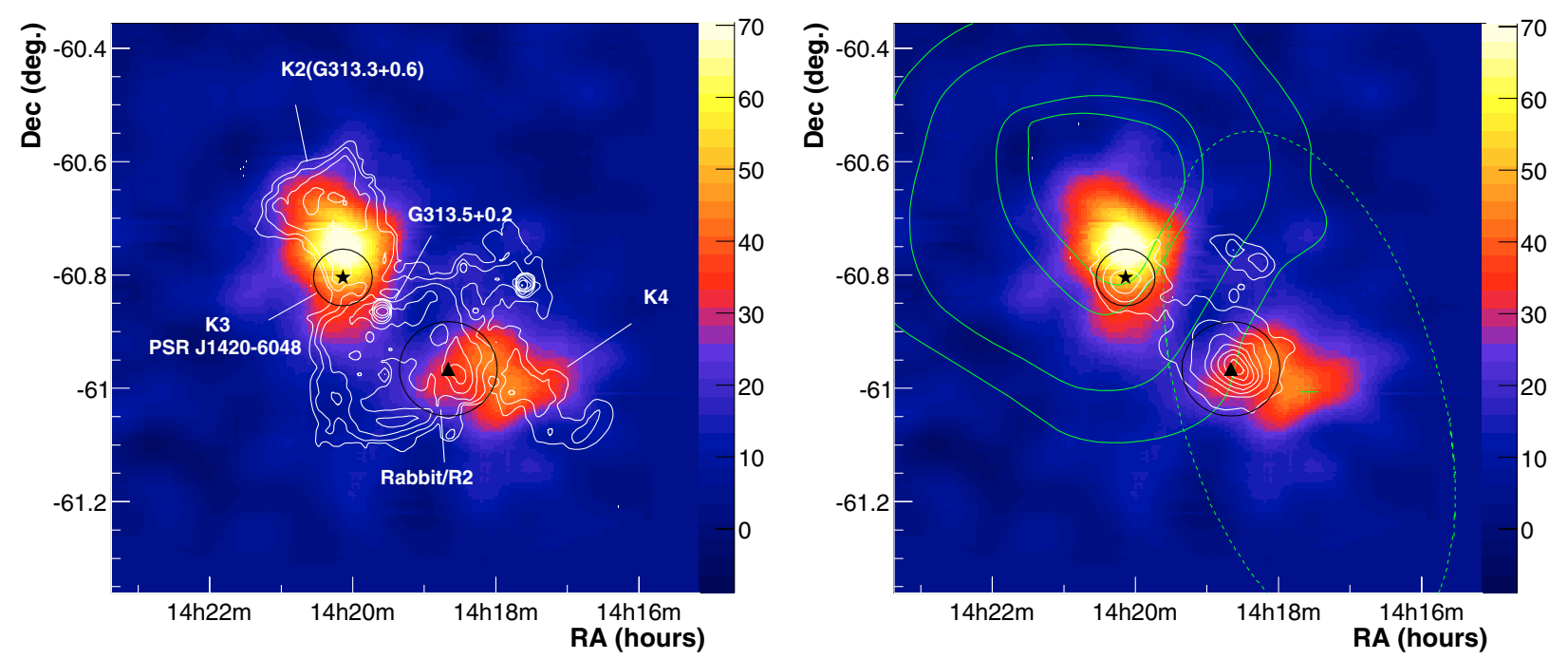

Fig. 3. HESS excess map smoothed with a Gaussian of $\sigma=2^{\prime}$. The black star and triangle mark the positions of the pulsar PSR J1420-6048 and the Rabbit (G313.3+0.1) R2 source (see text). Black circles around these two positions show the approximate extension of the X-ray diffuse emission for K3 (3') and the Rabbit (5') nebula (Ng et al. 2005). Left panel: white contours are from ATCA $20 \mathrm{~cm}$ high resolution images. The radio wings, K2 and K4 are clearly correlated with the HESS map, whereas there is no correspondence between the central shell, or the bright HII region, G313.5+0.2, and the VHE $\gamma$-ray emission. Right panel: ASCA GIS high energy band data are shown as white contours. Green contours show $>1 \mathrm{GeV}$ confidence levels $(50,68,95$ and 99\%) for 3EG J1420-6038; the green dashed ellipse and the green cross mark the 95\% error box and the postion of GeV J1417-6100. Note that although both EGRET sources are shown, they can not be considered as independent sources (see text).

improved understanding of instrumental responses and backgrounds, these two sources evolved to 3EG J1410-6147 (not shown in Fig. 3) and 3EG J1420-6038, respectively, in the third EGRET catalog (Hartman et al. 1999). The latter source exhibits a hard spectral index, $\Gamma=2.02 \pm 0.14$, and a flux above $100 \mathrm{MeV}$ of $73.8 \pm 12.1 \times 10^{-8} \mathrm{~cm}^{-2} \mathrm{~s}^{-1}$, similar to values reported for 3EG J1410-6147. Due to their small nominal distance as compared to the point spread function (PSF) of the instrument and the above-mentioned similarity, these sources are confused and were accordingly graded as "C". An analysis above $1 \mathrm{GeV}$ (Lamb \& Macomb 1997) of the EGRET data from the first 4 years of CGRO operation, where the higher energy of the $\gamma$-ray events results in a narrower PSF, yielded only one source in the Kookaburra region, GeV J1417-6100, with a flux $(>1 \mathrm{GeV})$ of $9.8 \pm 1.8 \times 10^{-8} \mathrm{~cm}^{-2} \mathrm{~s}^{-1}$. In Fig. 3, both 3EG J1420-6038 (confidence contours) and GeV J1417-6100 (error box and cross) are shown. HESS J1420-607 lies at an angular distance of $7.5^{\prime}\left(\theta_{95}^{\text {EGRET }} \sim 20^{\prime}\right)$ to the former, whereas the position reported for GeV J1417-6100 is at $4^{\prime}$ of

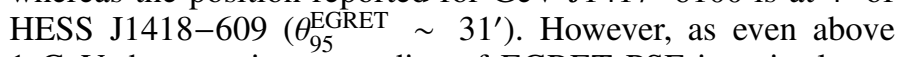
$1 \mathrm{GeV}$ the containment radius of EGRET PSF is quite large, GeV J1417-6100 and 3EG J1420-6038 share photons and can not be considered as independent sources. Another feature of the EGRET data is the indication of variability reported by Nolan et al. (2003) for 3EG J1420-6038. Comparing the estimated level of variability with the average for EGRET PWN candidates, these authors suggest the possible contribution of a PWN component to the $\gamma$-ray emission above $100 \mathrm{MeV}$ of 3EG J1420-6038.

\section{Discussion}

\subsection{Morphology: large offset nebulæ}

Following the multi-wavelength discussion of the previous section, the two discovered VHE sources are most plausibly associated to the two candidate PWNe, K3/PSR J1420-6049 and
Rabbit/R2. The extent of the TeV sources and their spatial coincidence with the radio wings, $\mathrm{K} 2$ and $\mathrm{K} 4$, as well as the nonthermal properties of the latter, strongly suggest that the wings are at least partly related to the two PWNe. This connection and the respective positions of $\mathrm{K} 3$ and the Rabbit on the edges of the two HESS sources imply in turn an asymmetric/offsetnebula type configuration, similar to that of the rapidly moving PWNe (or RPWNe), e.g. the Mouse nebula G359.23-0.3, or analogous to one-sided "crushed" nebulæ, e.g. Vela X or G18.0-0.7. Both of the latter objects have recently been associated to VHE sources, HESS J0836-456 (Aharonian et al. 2006b) and HESS J1825-137 (Aharonian et al. 2005c), respectively.

Given the distance estimate for PSR J1420-607, $d=5.6 d_{5.6} \mathrm{kpc}$, the measured $(2 \sigma)$ angular extension of HESS J1420-607 yields a relatively large nebular projected diameter of $11 d_{5.6}$ pc. For HESS J1418-609 and the Rabbit, the rough estimate of the distance of $\mathrm{R} 2, d \sim 5 d_{5} \mathrm{kpc}$, would imply a larger, but still comparable size of $14 d_{5} \mathrm{pc} \times 8 d_{5} \mathrm{pc}$. The implications of such large extensions will be discussed briefly in Sect. 4.3.

\subsection{Spectral energy distribution}

Figure 4 shows tentative broadband spectral energy distributions (SED) of the HESS sources, assuming their association with the radio/X-ray nebulæ and with the wings. X-ray spectra are plotted for overall nebulæ following the discussion in Sects. 3.1 and 3.2 (ASCA measurements of Roberts et al. 2001a), and can be significantly contaminated by the compact nebula hard emission. For the radio continuum, although the wings extend further than the TeV sources, the $S_{20 \mathrm{~cm}} \sim 1$ Jy flux of K2/K 3 and the total Rabbit+K4 flux, $S_{20 \mathrm{~cm}} \sim 0.8 \mathrm{Jy}$, are plotted. Given the discrepancy in spatial resolution between $\gamma$-ray observations with EGRET and HESS, we indicate the energy flux of 3EG J1420-6038 for both HESS sources. Assuming a pulsaronly origin first, the inferred conversion efficiency of spin-down 


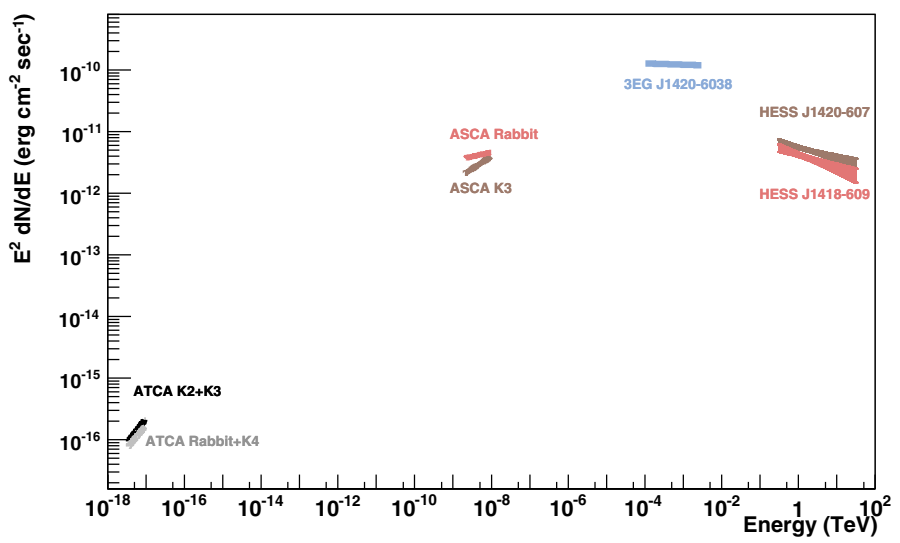

Fig. 4. Spectral energy distribution for HESS J1420-607/K3/K2 and HESS J1418-609/Rabbit/K4: TeV fitted spectra are shown as brown and red error boxes, respectively. The 3EG J1420-6038 energy flux is plotted in blue. X-ray spectra are given for overall nebulæ following the discussion in Sects. 3.1 and 3.2 (ASCA measurements of Roberts et al. 2001a), in brown and red for K3 and the Rabbit, respectively. For the radio continuum, although the wings could only be partly associated to the TeV sources, the $S_{20 \mathrm{~cm}} \sim 1$ Jy flux of K2/K3 (black), assuming the $\mathrm{K} 3$ spectral index $\alpha_{20 / 36}=-0.2$, and the total Rabbit $+\mathrm{K} 4$ flux, $S_{20 \mathrm{~cm}} \sim 0.8 \mathrm{Jy}$ (gray), are plotted. As the radio spectrum of $\mathrm{K} 4$ is poorly constrained $\left(\alpha_{20 / 36}=-1.2 \pm 0.5\right)$ the Rabbit nebula index $\alpha_{20 / 36}=-0.25$ has been used. Note that different integration radii are used (see text) and that the X-ray fluxes may be significantly contaminated by contribution from the compact nebular emissions.

power to pulsed luminosity in the $100 \mathrm{MeV}$ to $10 \mathrm{GeV}$ (assuming a typical Vela-like pulsed cutoff of $10 \mathrm{GeV}$ ) is $<1.6 \times 10^{-2} f$, with the unknown beaming factor $f=(\Delta \Omega / \beta) \%$ the ratio of the $\gamma$-ray beaming solid angle to the pulse duty cycle $\beta$, while the " $<$ " sign refers to the sharing of photons in the case of two unresolved overlapping EGRET sources. With a typical $f \sim 1$, the inferred conversion efficiency would then be of the same order of magnitude as that of the Vela (EGRET) pulsar for the same energy band. The other possibility, favoured by the indication of variability in EGRET data (see Sect. 3.3), is the contribution of a plerionic component in which case, given the similarity of the flux/spectra of the HESS sources, the overall EGRET flux would include contributions from both HESS J1420-607 and HESS J1418-609.

In a plerionic scenario, given the increasing order of synchrotron loss time-scales for X-ray, VHE, GeV and radio emitting electrons and the different integration radii for spectral measurements, the SEDs could reflect the synchrotron/inverse Compton (IC) emission of different populations of particles in different regions.

For the following discussion, a typical PWN field strength of $B \sim 10^{-5} B_{-5} \mathrm{G}$ is assumed and the Cosmic Microwave Background (CMBR) is chosen as dominant target photons. The synchrotron lifetime of parent electrons which produce $\gamma$-rays by IC scattering of these photons, with $E_{\mathrm{e}^{-}, \mathrm{IC}} \approx 20 E_{\mathrm{TeV}}^{1 / 2} \mathrm{TeV}$, is $\tau\left(E_{\mathrm{e}^{-}, \mathrm{IC}}\right) \approx 4.8 B_{-5}^{-2} E_{\mathrm{TeV}}^{-1 / 2} \mathrm{kyr}$. This yields, for the mean $\bar{E}_{\mathrm{TeV}} \sim 0.8 \mathrm{TeV}$ and $\bar{E}_{\mathrm{GeV}} \sim 0.5 \mathrm{GeV}$ energies, $\tau\left(E_{\mathrm{e}^{-}}\right) \sim 5$ and $\sim 200 \mathrm{kyr}$, respectively. Thus, whereas the electron lifetimes of VHE emitting electrons can be comparable to the ages of these two PWNe, the radio and GeV emitting lifetimes should be much longer. Only the $\mathrm{X}$-ray emitting lifetimes should be much shorter: $\tau\left(E_{\mathrm{e}^{-}, \mathrm{Syn}}\right)=1.2 B_{-5}^{-3 / 2} E_{\mathrm{keV}}^{-1 / 2} \mathrm{kyr}$, where $E_{\mathrm{e}^{-}, \text {Syn }}=70 B_{-5}^{-1 / 2} E_{\mathrm{keV}}^{1 / 2} \mathrm{TeV}$. The hard X-ray emitting electrons in the extended nebula would then correspond to freshly injected
Table 1. $\gamma$-ray luminosities, efficiencies and ages for the two PWNe as compared to G18.0-0.7/HESS J1825-137. Distances of 5.6, 5 and $4 \mathrm{kpc}$ have been used for HESS J1420-609, HESS J1418-607, and HESS J1825-137 respectively.

\begin{tabular}{lcccc}
\hline \hline Source & $\begin{array}{c}\dot{E} \\
10^{36} \mathrm{erg} / \mathrm{s}\end{array}$ & $\begin{array}{c}L_{\gamma} \\
10^{33} \mathrm{erg} / \mathrm{s}\end{array}$ & $\begin{array}{c}\epsilon_{\gamma} \\
\%\end{array}$ & $\begin{array}{c}\text { Age } \\
\mathrm{kyr}\end{array}$ \\
\hline K3/ & & & & \\
HESS J1420-609 & 10 & 51 & 0.51 & 13 \\
Rabbit/ & & & & \\
HESS J1418-607 & $\sim 5-30$ & 48 & $0.96-0.16$ & $1.6 ?$ \\
$\begin{array}{l}\text { G18.0-0.7/ } \\
\text { HESS J1825-137 }\end{array}$ & 2.8 & 100 & 3.6 & 21 \\
\hline
\end{tabular}

electrons by the pulsar wind shock. Upon advection away from the pulsar wind shock, the X-ray photon index should steepen towards the outer nebula as a result of cooling. However, the measured radio and possibly $\mathrm{GeV}$ emissions are expected to contain uncooled particles from the earliest stages of the pulsar injection. In contrast, those emitting at $\mathrm{TeV}$ energies (for pulsar ages $\tau_{\mathrm{c}} \gtrsim 10 \mathrm{kyr}$ ) should be composed mainly of cooled particles cumulated for up to 5-10 kyr. Hence, radio and GeV nebulæ should have larger sizes than that of the $\mathrm{TeV}$ nebula for evolved PWNe and the latter should in turn be larger than the hard X-ray diffuse emission. In this simple picture the difference between the VHE $\gamma$-ray and the X-ray spectral indices should be $\sim 0.5$. The measured $\Gamma_{\mathrm{TeV}} \sim 2.2$ (determined within a radius $\sim 10^{\prime}$ for both nebulæ), when compared to the X-ray measurements on smaller radii (within few arc-minutes), $\Gamma_{X} \sim 1.5-1.8$, are roughly consistent with this picture. One expects also a harder VHE spectrum relative to the total observed, if events are selected within the PSF around these two source origins. Such details will however be addressed in a future paper.

Realistically, the situation becomes more complicated when magnetic field variations in space/time, evolution of the pulsar spin-down luminosity, or other potential target photons, e.g. a cold dust component, are taken into account. A higher field strength in the early epochs would shorten the cooling timescales, whereas the IC electrons corresponding to dust IR targets have longer synchrotron lifetimes ( factor of two for $25 \mathrm{~K}$ targets).

In Table 1 , the $\gamma$-ray luminosities of the two sources are compared to the recently discovered TeV source HESS J1825-137. The latter, associated with G18.0-0.7 and the Vela-like pulsar PSR B1823-13, shows a similar offset morphology with an even larger TeV nebula $(D \sim 34 \mathrm{pc})$. As mentioned above, the TeV luminosity should reflect the emission of an accumulated population of particles injected into the nebula through the lifetime of the pulsar. For similar magnetic field configurations, the apparent $\gamma$-ray efficiency should be an increasing function of the nebula age for young and middle-aged PWN, and may reach large values when derived with respect to the present day spin-down luminosity. The values derived here follow this trend although they suffer from large uncertainties on distance and age measurements, especially for the Rabbit. For HESS J1420-609 and/or HESS J1418-607, they may have been significantly underestimated if the $\gamma$-ray emission continues into the EGRET domain.

\subsection{Extended one-sided nebulæ scenarios}

In the following discussion we adopt $D \sim 10 \mathrm{pc}$ for the spatial extent (projected diameter) of the two HESS sources. The large sizes of the TeV sources imply very high speeds for transporting 
the particles to the edges of the PWNe within their synchrotron lifetimes, e.g. $D / \tau\left(E_{\mathrm{e}^{-}, \text {IC }}\right) \sim 2000 \mathrm{~km} \mathrm{~s}^{-1}$. We examine briefly two scenarios where such large and asymmetric extents can be expected.

The Mouse nebula, G359.23-0.82, is a well studied example of RPWNe, i.e. an energetic pulsar interacting with its surrounding material: the supersonic pulsar's velocity confines the nebula through ram pressure resulting in a bow-shock structure (Gaensler et al. 2004) and an elongated nebula with the pulsar at its apex. The "tail" of the mouse contains shocked pulsar material convected away at very high flow velocities, typically fractions of light speed as shown by relativistic MHD simulations of Bucciantini et al. (2005). While X-ray exposures seem too short to reveal fine details for either PWN candidate ( $\mathrm{Ng}$ et al. 2005), the high resolution ATCA radio images of $\mathrm{K} 2 / \mathrm{K} 3$ and the Rabbit (Roberts et al. 2001b, 1999) show filamentary enhancements which could trace North-South or West-East motions, respectively. However, the fact that a bright part of the X-ray nebula is to the South of PSR J1420-6049 while the VHE emission lies mainly to its North argues against this interpretation for K3/HESS J1420-607. In the case of the Rabbit/HESS J1418-609, the uncollimated diffuse X-ray and VHE emissions may not be consistent with the RPWN scenario, either.

One-sided PWNe can also be produced in evolved systems in which the reverse shock from the surrounding SNR displaces the nebula (Reynolds \& Chevalier 1984). During the crushing phase, the nebula particles can be convected with speeds of $\sim 1000 \mathrm{~km} \mathrm{~s}^{-1}$ (van der Swaluw et al. 2001) away from the pulsar and feed an offset nebula, if initial asymmetries in the system (offset of the pulsar with respect to the expanding ejecta, density gradients around the birth site) yield a composite reverse shock with different arrival timescales to the PWN (Chevalier 1998). Simulations of (Blondin et al. 2001) show that for a symmetrical system and reasonable assumptions the start of inward motion of the reverse shock occurs at $\sim 1500 \mathrm{yr}$ and the crushing takes place on timescales of few thousand years, after which the expected ratio of the pulsar nebula radius to that of the SNR $R_{\text {PWN }} / R_{\text {SNR }} \sim 0.25$. The size of the undetected parent SNR for PSR J1420-6048, assuming an age $\tau \sim 13 \mathrm{kyr}$ and expansion in an environment of density $\sim$ few $10^{-1} \mathrm{~cm}^{-3}, R_{\text {SNR }} \sim 20-25 \mathrm{pc}$ as compared to the measured $R_{\mathrm{PWN}}$, would fit with the aforementioned simulations for HESS J1420-607. The offset morphology of Rabbit/HESS J1418-609 could also be explained through this scenario, provided that they constitute an evolved PWN, i.e. the age of the system is at least few thousand years. In this case the parent SNR again remains undetected.

\section{Conclusions}

Two extended VHE sources have been discovered in the wings of the Kookaburra complex, HESS J1420-609 and HESS J1418-607. They show similar $\gamma$-ray angular extensions $\left(\sim 3.3^{\prime}-3.4^{\prime}\right)$ and hard VHE $\gamma$-ray spectra. This discovery confirms the non-thermal nature of at least parts of the wings of the Kookaburra which overlap with the VHE emission and establishes their connection with the two X-ray PWN candidates, K3 and the Rabbit. Within the limits of available multiwavelength data, the SEDs of the two sources show also remarkable similarities and suggest analogous underlying objects and emission processes. The VHE $\gamma$-ray emission could most plausibly be explained by IC emission from these PWNe in an offsettype configuration near the energetic PSR J1420-6048, and the candidate pulsar in the Rabbit nebula. Given the poor spatial resolution of EGRET measurements, the unidentified source(s) 3EG J1420-6038/GeV J1417-6100 could possibly be related to either or both HESS sources through a PWN-type emission above $100 \mathrm{MeV}$. The EGRET flux may also contain pulsed emission from the pulsar associated with $\mathrm{K} 3$ and the candidate pulsar in the Rabbit. Future GLAST observations of these objects should be able to establish the status of such pulsed emission, resulting in a more certain multiwavelength interpretation of the HESS sources. The detection of the Rabbit pulsar, the morphology of the VHE and X-ray nebulæ, and the confirmation of a $\mathrm{GeV}-\mathrm{TeV}$ connection are important considerations that need to be addressed through further investigations.

Acknowledgements. The support of the Namibian authorities and of the University of Namibia in facilitating the construction and operation of HESS is gratefully acknowledged, as is the support by the German Ministry for Education and Research (BMBF), the Max Planck Society, the French Ministry for Research, the CNRS-IN2P3 and the Astroparticle Interdisciplinary Programme of the CNRS, the UK Particle Physics and Astronomy Research Council (PPARC), the IPNP of the Charles University, the South African Department of Science and Technology and National Research Foundation, and by the University of Namibia. We appreciate the excellent work of the technical support staff in Berlin, Durham, Hamburg, Heidelberg, Palaiseau, Paris, Saclay, and in Namibia in the construction and operation of the equipment. The authors wish to thank the referee, M. Roberts, for very useful comments on the multi-wavelength discussion.

\section{References}

Aharonian, F. A., et al. (HESS Collaboration) 2004, Nature, 432, 75 Aharonian, F. A., et al. (HESS Collaboration) 2005a, Science, 307, 1938 Aharonian, F. A., et al. (HESS Collaboration) 2005b, A\&A, 430, 865 Aharonian, F. A., et al. (HESS Collaboration) 2005c, A\&A, 442, L25 Aharonian, F. A., et al. (HESS Collaboration) 2006a, ApJ, 636, 777 Aharonian, F. A., et al. (HESS Collaboration) 2006b, A\&A, 448, L43 Aharonian, F. A., et al. (HESS Collaboration) 2006c, A\&A, submitted D’Amico, N., Kaspi, V. M., Manchester, R. N., et al. 2001, ApJ, 552, L45 Bernlöhr, K., Carrol, O., Cornils, R., et al. 2003, APh, 20, 111 Blondin, J. M., Chevalier, R. A., \& Frierson, D. M. 2001, ApJ, 563, 806 Bucciantini, N., Amato, E., \& Del Zanna, L. 2005 A\&A, 434, 189 Case, G., \& Bhattacharya, D. 1999, ApJ, 521, 246

Chevalier, R. A. 1998, Mem. Soc. Astron. It., 69, 977

Cordes, J. M., \& Lazio, T. J. W. 2002, preprint [arXiv: astro-ph/0207156] Funk, S., Hermann, G., Hinton, J., et al. 2004, APh, 22, 285

Gaensler, B. M., Jones, D. H., \& Stappers, B. W. 2002, ApJ, 580, L137 Gaensler, B. M., van der Swaluw, E., Camilo, F., et al. 2004, ApJ, 616, 383 Gotthelf, E. V. 2003, ApJ, 591, 361

Hartman, R. C., Bertsch, D. L., Bloom, S. D., et al. 1999, ApJS, 123, 79H Hinton, J. A. 2004, NewAR, 48, 331

Hinton, J. A., Berge, D., \& Funk, S. 2005, Proc. Cherenkov 2005 (Palaiseau) Lamb, R. C., \& Macomb, D. J. 1997, ApJ, 488, 872

Nolan, P. L., Tompkins, W. F., Grenier, I. A., \& Michelson, P. F. 2003, ApJ, 597, 615

Ng, C.-Y., Roberts, M. S. E., \& Romani, R. W., 2005, ApJ, 627, 904

Piron, F., Djannati-Ataï, A., Punch, M., et al. 2001, A\&A, 374, 895

Reynolds, S. P., \& Chevalier, R. A. 1984, ApJ, 278, 630

Roberts, M. S. E., \& Romani, R. W. 1998, ApJ, 496, 827

Roberts, M. S. E., Romani, R. W., Johnston, S., \& Green, A. 1999, ApJ, 515, 712

Roberts, M. S. E., Romani, R. W., \& Kawai, N. 2001a, ApJS, 133, 451 Roberts, M. S. E., Romani, R. W., \& Johnston, S. 2001b, ApJ, 561, L187 Taylor, J. H., \& Cordes, J. M. 1993, ApJ, 411, 674

Thompson, D. J., Bertsch, D. L., Dingus, B. L., et al. 1995, ApJS, 101, 259 Thompson, D. J., Bertsch, D. L., Dingus, B. L., et al. 1996, ApJS, 107, 227 van der Swaluw, E., Achterberg, A., Gallant, Y. A., \& Toth, G. 2001, A\&A, 380, 309

Vincent, P., et al. 2003, in Proceedings of the 28th International Cosmic Ray Conference (Tsukuba), 1, 2887

1 Max-Planck-Institut für Kernphysik, PO Box 103980, 69029 Heidelberg, Germany

2 Yerevan Physics Institute, 2 Alikhanian Brothers St., 375036 Yerevan, Armenia

3 Centre d'Étude Spatiale des Rayonnements, CNRS/UPS, 9 Av. du Colonel Roche, BP 4346, 31029 Toulouse Cedex 4, France 
${ }^{4}$ Universität Hamburg, Institut für Experimentalphysik, Luruper Chaussee 149, 22761 Hamburg, Germany

5 Institut für Physik, Humboldt-Universität zu Berlin, Newtonstr. 15, 12489 Berlin, Germany

e-mail: Stefan.Funk@mpi-hd.mpg.de

${ }^{6}$ LUTH, UMR 8102 du CNRS, Observatoire de Paris, Section de Meudon, 92195 Meudon Cedex, France

7 University of Durham, Department of Physics, South Road, Durham DH1 3LE, UK

8 Unit for Space Physics, North-West University, Potchefstroom 2520, South Africa

9 Laboratoire Leprince-Ringuet, IN2P3/CNRS, École Polytechnique, 91128 Palaiseau, France

10 APC, UMR 7164 (CNRS, Université Paris VII, CEA, Observatoire de Paris), 11 place Marcelin Berthelot, 75231 Paris Cedex 05, France

e-mail: djannati@apc.univ-paris7.fr

11 Dublin Institute for Advanced Studies, 5 Merrion Square, Dublin 2, Ireland

12 Landessternwarte, Universität Heidelberg, Königstuhl, 69117 Heidelberg, Germany
13 Laboratoire de Physique Théorique et Astroparticules, IN2P3/CNRS, Université Montpellier II, CC 70, Place Eugène Bataillon, 34095 Montpellier Cedex 5, France

14 DAPNIA/DSM/CEA, CE Saclay, 91191 Gif-sur-Yvette Cedex, France

15 Laboratoire d'Astrophysique de Grenoble, INSU/CNRS, Université Joseph Fourier, BP 53, 38041 Grenoble Cedex 9, France

16 Institut für Astronomie und Astrophysik, Universität Tübingen, Sand 1, 72076 Tübingen, Germany

17 Laboratoire de Physique Nucléaire et de Hautes Énergies, IN2P3/CNRS, Universités Paris VI \& VII, 4 place Jussieu, 75252 Paris Cedex 5, France

18 Institute of Particle and Nuclear Physics, Charles University, V Holesovickach 2, 18000 Prague 8, Czech Republic

19 Institut für Theoretische Physik, Lehrstuhl IV: Weltraum und Astrophysik, Ruhr-Universität Bochum, 44780 Bochum, Germany

20 University of Namibia, Private Bag 13301, Windhoek, Namibia

21 Universität Erlangen-Nürnberg, Physikalisches Institut, ErwinRommel-Str. 1, 91058 Erlangen, Germany

22 European Associated Laboratory for Gamma-Ray Astronomy, jointly supported by CNRS and MPG 